\title{
Cytotoxicity activity of geldanamycin derivatives against various cancer cell lines
}

\author{
Thongchai Taechowisan ${ }^{1 *}$, Tipparat Samsawat ${ }^{1}$, Winyou Puckdee ${ }^{1}$, Waya S. Phutdhawong ${ }^{2}$ \\ ${ }^{1}$ Department of Microbiology, Faculty of Science, Silpakorn University, Nakhon Pathom 7300, Thailand. \\ ${ }^{2}$ Department of Chemistry, Faculty of Science Silpakorn University, Nakhon Pathom 7300, Thailand.
}

\section{ARTICLE INFO \\ Received on: 12/10/2019 \\ Accepted on: 02/04/2020 \\ Available online: 05/06/2020}

\section{Key words:}

Anticancer activity, cancer cell lines, cytotoxicity activity, solubility, tryptamine-geldanamycin hybrids.

\begin{abstract}
Geldanamycin (1) was isolated as a major compound from Streptomyces zerumbet W14. It was then used as a precursor to synthesize two new geldanamycins: 17-(tryptamine)-17-demethoxygeldanamycin (2) and 17-(5'-methoxytryptamine)17-demethoxygeldanamycin (3). The cytotoxicity activity of these two new compounds was evaluated and compared with the cytotoxicity of compound $\mathbf{1}$. Cytotoxicity activity was evaluated against a normal cell line, and three cancer cell lines using an 3-(4, 5-Dimethylthiazol-2-yl)-2, 5-diphenyltetrazolium bromide (MTT) colorimetric assay. The solubility of these compounds was also determined. The solubility of compounds $\mathbf{2}$ and $\mathbf{3}$ in water was 290.69 and $348.18 \mu \mathrm{M}$, higher than that of compound $\mathbf{1}$ by about 1.91 and 2.29 times, respectively. Compounds 2 and 3 showed moderate cytotoxic activity on Vero and human cervical carcinoma cells with IC50 values of $>200.00 \mu \mathrm{g} /$ $\mathrm{ml}$. The strongest cytotoxicity of compound $\mathbf{3}$ was observed in human breast carcinoma cells (MCF-7) and human hepatocellular carcinoma cell line (HepG2) cells with IC50 values of 82.50 and $114.35 \mu \mathrm{g} / \mathrm{ml}$, respectively, while the IC50 values of compound 2 against MCF-7 and HepG2 cells were 105.62 and $124.57 \mu \mathrm{g} / \mathrm{ml}$, respectively. The findings showed that these new geldanamycin derivatives exhibited selective cytotoxicity toward some cancer cells at a lower concentration. Therefore, future studies on these compounds could be useful for the management of some cancers.
\end{abstract}

\section{INTRODUCTION}

Geldanamycin was the first benzoquinone ansamycin antibiotic that exhibited anticancer activity by inhibiting kinase folding by the Hsp90 chaperone complex in a wide range of cancers (Whitesell et al., 1994). The blockage of Hsp90 function induced the proteasome-dependent degradation of cancer relevant target proteins, known as client proteins (Mimnaugh et al., 1996). Despite its anticancer potential in vitro, the clinical use of geldanamycin has not been considered due to several limitations (Supko et al., 1995). First, it exhibited severe hepatotoxicity and nephrotoxicity at therapeutic recommended doses in animal models, limiting effective doses, and thus was

\footnotetext{
"Corresponding Author

Thongchai Taechowisan, Department of Microbiology, Faculty of Science, Silpakorn University, Nakhon Pathom 7300, Thailand.

E-mail:tewson84@hotmail.com
}

unacceptable as a therapeutic profile. This toxicity seemed to be caused by metabolisms of the benzoquinone moiety. Second, geldanamycin is metabolically unstable and poorly soluble in water. Accordingly, variants of geldanamycin have been developed, most notably by altering the quinone ring structure, which have led to improvements in tolerance, potency, metabolic stability, and water solubility (Le Brazidec et al., 2004). Although, the synthesized series of geldanamycin derivatives to make new types of Hsp90 inhibitor with weak toxicity and high efficiency have been attempting (Jurczyszyn et al., 2014; Lee, 2018; Lin et al., 2015; Vasilevskaya et al., 2003). However, there was a limit number of water solubility of these geldanamycin derivatives.

Tryptamine was derived by the decarboxylation of tryptophan. Tryptamine has been used in the past as a neurotransmitter and neuromodulator, vasoconstrictor, and vasodilator, and as antibacterial, antifungal, antiviral, antioxidant, and anti-inflammatory agent (Kousara et al., 2017). 
Its modification has led to many compounds of pharmacological importance. Recently, tryptamine-based compounds have been synthesized as anticancer agents (Guo et al., 2019; Malik et al., 2019; Wang et al., 2016). It has been an effective tool for improving water solubility, biological potency, and pharmacokinetic properties of many natural products (Kousara et al., 2017). According to these effects, the invention of tryptamine-geldanamycin hybrids has been designed. The $\mathrm{C} 17$ methoxyl of the geldanamycin molecule can allow for various nucleophiles to be introduced. Thus, geldanamycin from the beginning has been a popular template for semi-synthetic analogs (Lin et al., 2015; Modi et al., 2011; Supko et al., 1995; Tian et al., 2004; Wrona et al., 2010). In this study, two new geldanamycin derivatives were synthesized in form tryptaminegeldanamycin hybrids by substituting with tryptamine and 5-methoxytryptamine that consist of heterocyclic and strong polar groups in the 17-position to improve the pharmacokinetic properties. To our knowledge, this is the first report to describe the screening of tryptamine-geldanamycin hybrids as anticancer agents. The aim of the present study was to evaluate the anticancer activity of the synthesized tryptamine-geldanamycin hybrids against some cancer cell lines: [human breast carcinoma cells (MCF-7); human cervical carcinoma cells (HeLa); and human hepatocellular carcinoma cells (HepG2)] and their water solubility was also determined.

\section{MATERIALS AND METHODS}

\section{Isolation and cultivation of endophytic actinomycete}

Actinomycete strain W14 was isolated from the rhizome tissue of Zingiber zerumbet (L.) Smith, collected from Chanthaburi province, Thailand. The samples were used to isolate the endophytic actinomycetes by surface-sterilization technique and validation of surface sterilization was performed as described in the previous studies (Coombs and Franco, 2003; Taechowisan et al., 2017). Strain W14 was selected and identified using morphological, cultural, physiological, and biochemical characteristics, chemotaxonomy and 16S rDNA sequencing (Taechowisan and Lumyong, 2003; Taechowisan et al., 2019). This strain was grown on ISP-2 agar at $30^{\circ} \mathrm{C}$ for 14 days, and then the culture medium was cut into small pieces that were extracted with ethyl acetate $(3 \times 500 \mathrm{ml})$. This organic solvent was taken to dryness under rotary evaporation. The solid was separated by column chromatography using silica gel 60 (Merck, $0.040-0.063 \mathrm{~mm}$ ), and $30 \%, 50 \%, 75 \%$, and $100 \%$ of ethyl acetate in hexane as the eluent to give 17 main fractions (F1-F17). Fraction F13 (30.3 mg) gave a very prominent single spot of pure compound on thin-layer chromatography (TLC) and was used to investigate on nuclear magnetic resonance (NMR) spectroscopy. The spectral data revealed this compound to be geldanamycin $\left(\mathrm{C}_{29} \mathrm{H}_{40} \mathrm{~N}_{2} \mathrm{O}_{9}\right)$ (1).

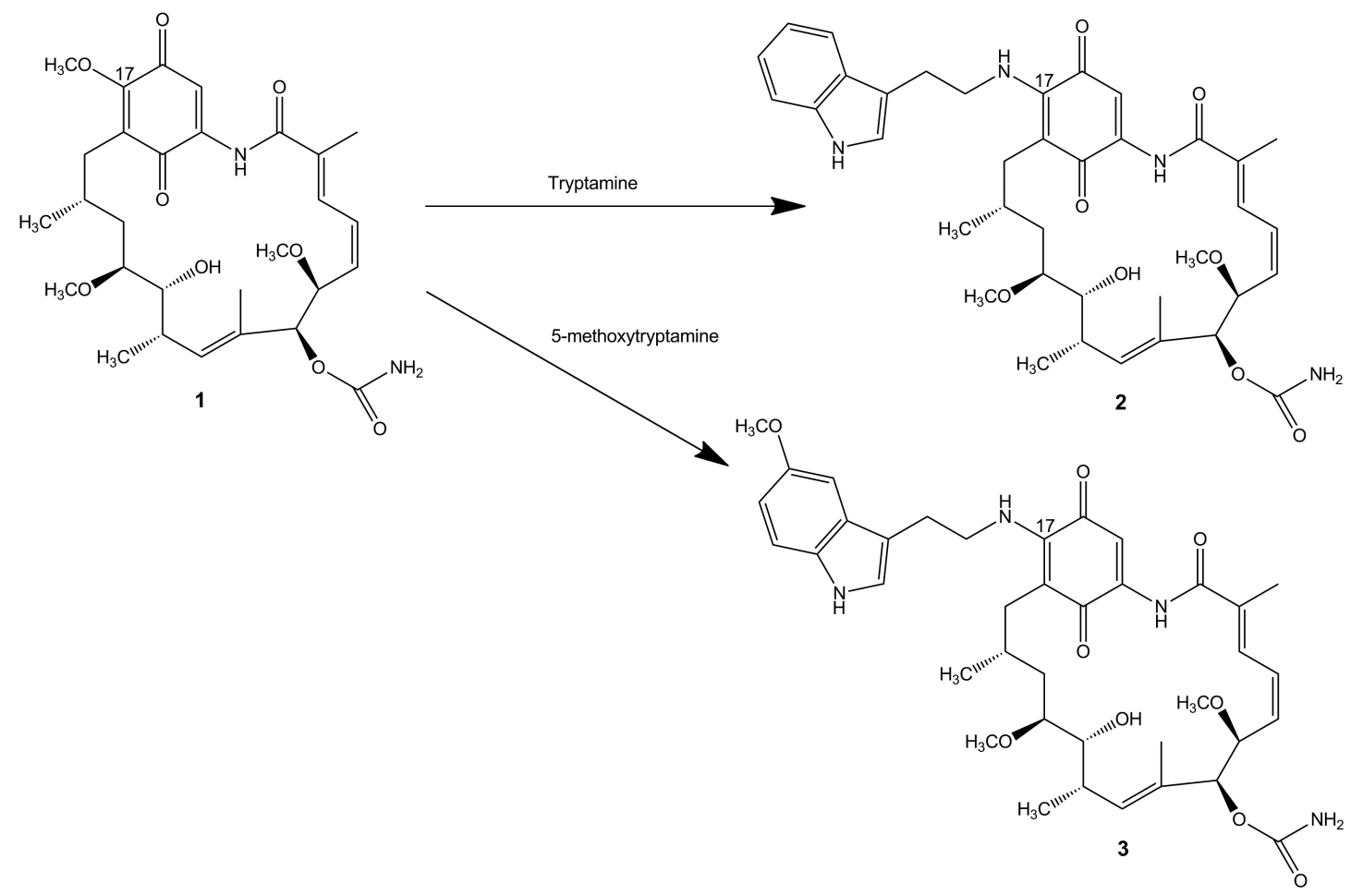

Figure 1. Geldanamycin chemical structure (1) and structures and synthesis of two derivatives; 17-(tryptamine)-17demethoxygeldanamycin (2) and 17-(5'-methoxytryptamine)-17-demethoxygeldanamycin (3). 


\section{Synthesis of geldanamycin derivatives}

All procedures and experiments described were geladanamycin derivative synthesis. Geladanamycin derivatives; 17-(tryptamine)17-demethoxygeldanamycin $\quad\left(\mathrm{C}_{38} \mathrm{H}_{48} \mathrm{~N}_{4} \mathrm{O}_{8}\right) \quad$ (2) and 17-(5'-methoxytryptamine)-17-demethoxygeldanamycin $\left(\mathrm{C}_{39} \mathrm{H}_{50} \mathrm{~N}_{4} \mathrm{O}_{9}\right)$ (3) were synthesized from geldanamycin (Fig. 1). To a solution of geldanamycin $(0.84 \mathrm{~g}, 100 \mathrm{mmol})$ in dichloromethane $(15 \mathrm{ml})$ at $25^{\circ} \mathrm{C}$, tryptamine $(0.29 \mathrm{~g}, 150 \mathrm{mmol})$ (Sigma-Aldrich) or 5'-methoxytryptamine $(0.36 \mathrm{~g}, 150 \mathrm{mmol})$ (Sigma-Aldrich) was added. The reaction mixture was stirred at $25^{\circ} \mathrm{C}$ for 30 minutes before the addition of saturated aqueous $\mathrm{CaCl}_{2}(5 \mathrm{ml})$. The organic layer was removed and washed with saturated $\mathrm{CaCl}_{2}$ solution $(3 \times 5 \mathrm{ml})$ and dried $\left(\mathrm{Na}_{2} \mathrm{SO}_{4}\right)$. The mixture was filtered over Celite, rinsed with ethyl acetate, and concentrated under reduced pressure to give a dark purple solid. Purification by flash chromatography (silica, $60 \%$ ethyl acetate/ hexanes) affords compound $2(1.01 \mathrm{~g}, 97 \mathrm{mmol}, 96.8 \%)$ or compound 3 (1.03 g, 95 mmol, 94.7\%).

\section{Water solubility test}

The water solubility of geldanamycin and its derivatives was carried out by UV-spectroscopic assay in 96-well plates and measured using a SpectraMax Plus plate reader (Molecular Devices Sunnyvale, CA) as described by Hoelke et al. (2009). Briefly, the compounds were solubilized in water at various concentrations and incubated on a shaker deck at $30^{\circ} \mathrm{C}$ for 30 minutes. The samples were filtered and $200 \mu$ of the samples were transferred to 96 -well plates. The absorbance was measured at 450 $\mathrm{nm}$ for compound $\mathbf{1}$ and at $570 \mathrm{~nm}$ for compounds $\mathbf{2}$ and $\mathbf{3}$. The calibration of UV-spectroscopic measurements for solubility of each compound was plotted against the concentrations. The stable part of the optical density change was ascribed to water solubility of the compounds. A check of the photometric linearity of the UV plate reader using solutions of $\mathrm{K}_{2} \mathrm{Cr}_{2} \mathrm{O}_{7}$ at variable concentrations led to a linear range of the instrument from 0.003 to 3.5 absorption units for wavelengths $350 \mathrm{~nm}$.

\section{MTT assay for cytotoxicity activity}

A normal cell line [African green monkey kidney cells (Vero)] and three cancer cell lines (MCF-7; HeLa; HepG2) were obtained from the Korean Cell Line Bank (Seoul, Korea). These cells were grown at $37^{\circ} \mathrm{C}$ in Dulbecco's modified eagle's medium (DMEM) supplement with 10\% fetal bovine serum (FBS), penicillin (100 units/ml), and streptomycein sulfate (100 $\mu \mathrm{g} / \mathrm{ml}$ ) in a humidified atmosphere of $5 \% \mathrm{CO}_{2}$. Cytotoxicity studies were performed on a 96-well plate. The cells were mechanically scraped and plated $2 \times 10^{5}$ per well containing 100 $\mu 1$ of DMEM medium with $10 \%$ FBS and incubated overnight. The purified compound was dissolved in dimethylsulfoxide for stock solution. Cells were incubated with purified compound at increasing concentrations $(1.5625,3.125,6.25,12.5,25,50$, 100 , and $200 \mu \mathrm{l} / \mathrm{ml}$ ) in FBS-free medium for 24 hours. The dimethyl sulfoxide (DMSO) concentrations in all assays did not exceed $0.1 \%$. Cells were washed once before adding $50 \mu \mathrm{l}$ FBS-free medium containing $5 \mathrm{mg} / \mathrm{ml} \mathrm{3-(4,5-Dimethylthiazol-}$ 2-yl)-2, 5-diphenyltetrazolium bromide (MTT). After 4 hours of inoculation at $37^{\circ} \mathrm{C}$, the medium was discarded and the formazan blue, which formed in the cells, was dissolved in $50 \mu \mathrm{l}$ DMSO. The optical density was measured at $570 \mathrm{~nm}$. Consecutively, measurements for concentration required for $50 \%\left(\mathrm{IC}_{50}\right)$ inhibition were noted. Cell viability percent was calculated using the formula given below:

$$
\begin{aligned}
& \text { Percentage }(\%) \text { of cell viability }=A_{570} \text { of treated cells } \\
& A_{570} \text { of control cells } \\
& \times 100
\end{aligned}
$$

The graph was plotted with the $Y$-axis showing the percentage of viability of cells and $X$-axis showing the compound concentration.

The therapeutic index was calculated as the ratio of $\mathrm{IC}_{50}$ of normal cells to $\mathrm{IC}_{50}$ of cancer cells.

\section{Statistical analysis}

Values are expressed as means \pm standard deviation (SD) of three experiments. The SPSS v.16.0 software (SPSS Inc., Chicago, IL) was used for data analysis. Comparisons between two groups were analyzed using the two-tailed Dunnett $t$-tests treated compound 1 as a control group. A $p$-value $<0.05$ is considered as statistically significant.

\section{RESULTS}

The mass spectral data of geldanamycin and geldanamycin derivatives were carried out by ${ }^{1} \mathrm{H}-\mathrm{NMR},{ }^{13} \mathrm{C}-\mathrm{NMR}$ as following.

Compound (1): The infrared (IR) spectrum displayed characteristic absorption bands of $\mathrm{NH}$ and $\mathrm{OH}$ stretches at n $3,478,3,440,3,336$, and $3,297 \mathrm{~cm}^{-1}, \mathrm{CH}$ stretch in $\mathrm{CH}_{3}$ and

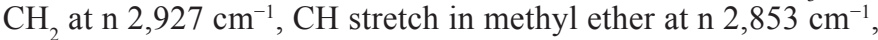
$\mathrm{C}=\mathrm{O}$ stretch in $\mathrm{OCONH}_{2}$ at $\mathrm{n} 1,729 \mathrm{~cm}^{-1}, \mathrm{C}=\mathrm{O}$ stretch in a,bunsaturated amide at $\mathrm{n} 1,701 \mathrm{~cm}^{-1}$ and $\mathrm{C}=\mathrm{O}$ stretches in quinone at $\mathrm{n} 1,675$ and $1,652 \mathrm{~cm}^{-1}$ (Fig. 2). The MS gave a $[\mathrm{M}+\mathrm{Na}]^{+}$ion at $\mathrm{m} / z 583.2571$ (Fig. 3a) which corresponded to the molecular formula $\mathrm{C}_{29} \mathrm{H}_{40} \mathrm{~N}_{2} \mathrm{O}_{9}$ for the compound indicating eleven double bond equivalents in the molecule. The structure was fully elucidated by ${ }^{1} \mathrm{H}-\mathrm{NMR},{ }^{13} \mathrm{C}-\mathrm{NMR}$ spectroscopy, DEPT-135, and 2D-NMR spectral studies. The spectral data revealed the compound 1 to be geldanamycin. Its ${ }^{1} \mathrm{H}-\mathrm{NMR}$ and ${ }^{13} \mathrm{C}-\mathrm{NMR}$ spectral data of which were in good agreement with those of geldanamycin (Table 1) previously reported by Ōmura et al. (1979) and Qin and Panek (2008).

Compound (2): The MS gave a $[\mathrm{M}+\mathrm{Na}]^{+}$ion at $\mathrm{m} / \mathrm{z}$ 711.3384 (Fig. 3b) which corresponded to the molecular formula $\mathrm{C}_{38} \mathrm{H}_{48} \mathrm{~N}_{4} \mathrm{O}_{8}$. The structure was fully elucidated by ${ }^{1} \mathrm{H}-\mathrm{NMR}$, ${ }^{13} \mathrm{C}$-NMR spectroscopy, DEPT-135, and 2D NMR spectral studies as shown in Table 2. 


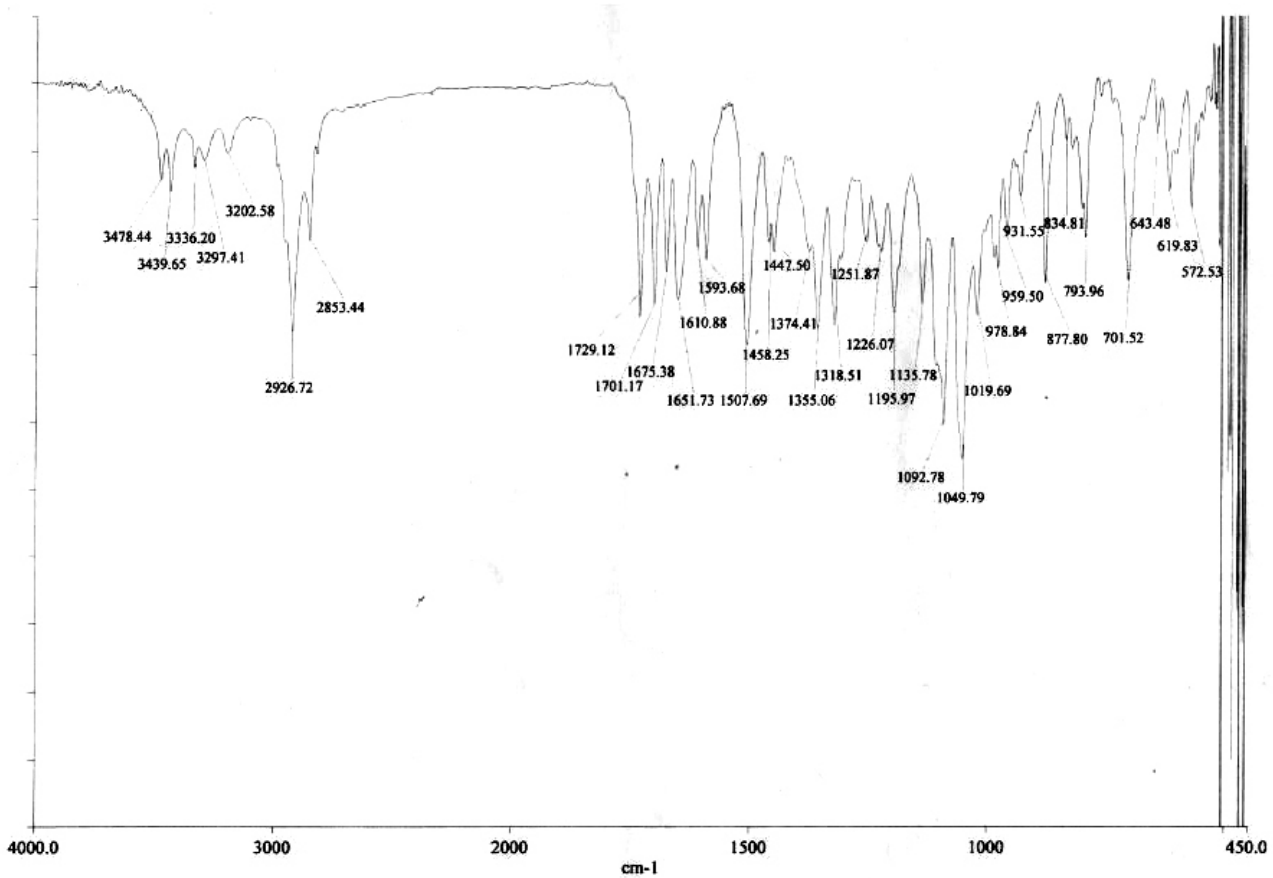

Figure 2. IR spectrum of compound $\mathbf{1}$.
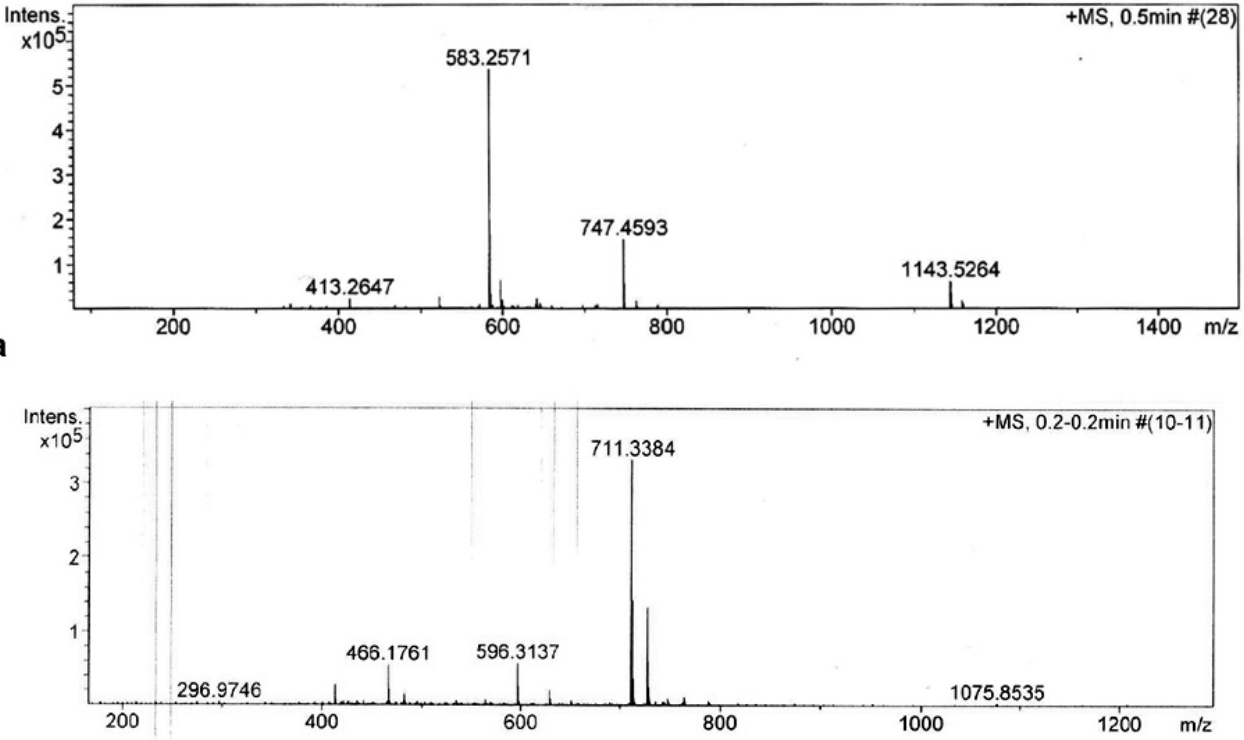

b

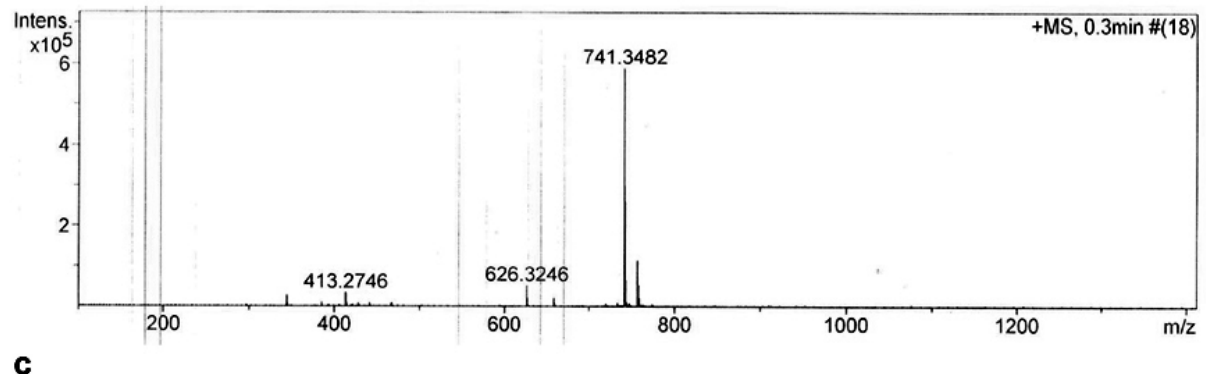

Figure 3. Mass spectrum of compounds $\mathbf{1}$ (a), 2 (b) and $\mathbf{3}$ (c). 
Table 1. Comparison of the spectral data of the compound $\mathbf{1}$ and GDA ${ }^{\mathrm{a}}$.

\begin{tabular}{|c|c|c|c|c|c|c|c|}
\hline No. & $\delta_{\mathrm{C}}$ compound 1 & $\delta_{\mathrm{C}}$ GDA & $\delta_{\mathrm{H}}$ compound 1 & $\delta_{\mathrm{H}}$ GDA & HMBC $(\mathbf{H} \rightarrow \mathbf{C})$ & COSY & NOESY \\
\hline 1 & $169.7 \mathrm{C}$ & 169.1 & - & - & - & - & - \\
\hline 2 & $133.2 \mathrm{C}$ & 133.2 & - & - & - & - & - \\
\hline 2-Me & $12.8 \mathrm{CH}_{3}$ & 12.2 & $1.93 \mathrm{~s}$ & $1.91, \mathrm{~s}$ & $1,2,3,4$ & - & - \\
\hline 3 & $128.7 \mathrm{CH}$ & 128.4 & $6.95 \mathrm{~d}$ & $6.95, \mathrm{~d}$ & - & 4 & $\mathrm{NH}, 4,6,7$ \\
\hline 4 & $126.3 \mathrm{CH}$ & 125.7 & $6.58 \mathrm{t}$ & $6.56, \mathrm{t}$ & 2,6 & 3,5 & 3,5 \\
\hline 5 & $138.7 \mathrm{CH}$ & 137.8 & $5.81 \mathrm{br}$ & $5.80, \mathrm{t}$ & - & 4,6 & 4,6 \\
\hline 6 & $82.3 \mathrm{CH}$ & 81.6 & $4.36 \mathrm{~d}(7.6)$ & $4.34, \mathrm{~d}$ & 4, 6-OMe & 5,7 & $3,5,7$ \\
\hline 6-OMe & $57.1 \mathrm{CH}_{3}$ & 56.0 & $3.24 \mathrm{~s}$ & $3.22, \mathrm{~s}$ & 6 & - & - \\
\hline 7 & $81.1 \mathrm{CH}$ & 80.6 & $4.88 \mathrm{br}$ & $4.86, \mathrm{br}$ & $5,7-\mathrm{OCONH}_{2}, 9,8-\mathrm{Me}$ & 6 & $3,6,9$ \\
\hline 7-OCONH & $156.6 \mathrm{C}$ & 156.0 & - & 6.45, br & - & - & - \\
\hline 8 & $129.1 \mathrm{C}$ & 132.6 & - & - & - & - & - \\
\hline 8-Me & $13.0 \mathrm{CH}_{3}$ & 12.5 & $1.62 \mathrm{~s}$ & $1.61, \mathrm{~s}$ & 7,9 & - & 10 \\
\hline 9 & $132.4 \mathrm{CH}$ & 131.9 & $5.50 \mathrm{~d}(8.5)$ & $5.51, \mathrm{~d}$ & 7, 8-Me & 10 & 7 \\
\hline 10 & $32.6 \mathrm{CH}$ & 32.1 & 2.56 & $3.61, \mathrm{~m}$ & - & $9,10-\mathrm{Me}$ & 8-Me, 10-Me, 11, 12 \\
\hline 10-Me & $23.4 \mathrm{CH}_{3}$ & 23.3 & $0.75 \mathrm{~d}(6.8)$ & $0.97, \mathrm{~d}$ & $9,10,11$ & 10 & $10,11,12$ \\
\hline 11 & $72.4 \mathrm{CH}$ & 71.9 & $3.09 \mathrm{br}$ & $3.29, \mathrm{~s}$ & $10-\mathrm{Me}$ & - & $10,10-\mathrm{Me}, 13,14$ \\
\hline $11-\mathrm{OH}$ & - & - & - & - & - & - & - \\
\hline 12 & $80.7 \mathrm{CH}$ & 80.2 & $3.09 \mathrm{br}$ & $3.07, \mathrm{~m}$ & 12-OMe & 13 & $10,10-\mathrm{Me}, 13,14$ \\
\hline 12-OMe & $56.5 \mathrm{CH}_{3}$ & 56.6 & $3.23 \mathrm{~s}$ & $3.23, \mathrm{~s}$ & 12 & - & - \\
\hline 13 & $31.3 \mathrm{CH}_{2}$ & 31.0 & $1.45 \mathrm{br}$ & $1.45, \mathrm{~m}$ & 14-Me & 12,14 & $11,12,14$ \\
\hline 14 & $27.1 \mathrm{CH}$ & 26.6 & $1.93 \mathrm{~s}$ & $1.91, \mathrm{br}$ & 12,16 & $13,14-\mathrm{Me}, 15 \mathrm{a}, 15 \mathrm{~b}$ & 13 \\
\hline 14-Me & $23.9 \mathrm{CH}_{3}$ & 23.0 & $0.97 \mathrm{br}$ & $0.76, \mathrm{~d}$ & 14,15 & 14 & - \\
\hline $15 \mathrm{a}$ & $32.2 \mathrm{CH}_{2}$ & 31.7 & $2.43 \mathrm{dd}(12.5,9.9)$ & $2.42, \mathrm{~m}$ & $13,14,16,17,21$ & $14,15 b$ & - \\
\hline $15 b$ & & 31.7 & $2.18 \mathrm{dd}(12.5,4.8)$ & & $13,14,16,17,21$ & $14,15 \mathrm{a}$ & - \\
\hline 16 & $128.7 \mathrm{C}$ & 128.1 & - & - & - & - & - \\
\hline 17 & $156.9 \mathrm{C}$ & 156.4 & - & - & - & - & - \\
\hline 17-OMe & $61.6 \mathrm{CH}_{3}$ & 61.0 & $3.96 \mathrm{~s}$ & $3.93, \mathrm{~s}$ & 17 & - & - \\
\hline 18 & $184.3 \mathrm{C}$ & 183.6 & - & - & - & - & - \\
\hline 19 & $111.3 \mathrm{CH}$ & 110.9 & $7.04 \mathrm{~s}$ & $7.02, \mathrm{~s}$ & - & - & - \\
\hline 20 & $140.1 \mathrm{C}$ & 139.6 & - & - & - & - & - \\
\hline 21 & $183.6 \mathrm{C}$ & 183.1 & - & - & - & - & - \\
\hline $\mathrm{NH}$ & - & - & $9.18, \mathrm{NH}$, br & $9.14, \mathrm{NH}$, br & $1,19,21$ & - & 3 \\
\hline
\end{tabular}

${ }^{a}$ GDA, geldanamycin (Ōmura et al., 1979).

Compound (3): The MS gave a $[\mathrm{M}+\mathrm{Na}]^{+}$ion at $m / z$ 741.3482 (Fig. 3c) which corresponded to the molecular formula $\mathrm{C}_{39} \mathrm{H}_{50} \mathrm{~N}_{4} \mathrm{O}_{9}$. The structure was fully elucidated by ${ }^{1} \mathrm{H}-\mathrm{NMR}$, ${ }^{13} \mathrm{C}$-NMR spectroscopy, DEPT-135, and 2D NMR spectral studies as shown in Table 2. The detailed comparison of spectral data of compounds $\mathbf{3}$ and $\mathbf{1}$ is shown in Table 3.

The water solubility of geldanamycin (1) was found to be $151.78 \mu \mathrm{M}$ (Table 4). In contrast, the solubility of its derivatives (2 and 3) in water was 290.69 and $348.18 \mu \mathrm{M}$, respectively, about 1.91 and 2.29 times, respectively, higher than that of geldanamycin. These data suggest that the attachment of a tryptamine moiety to geldanamycin at the $\mathrm{C} 17$ position greatly enhanced its water solubility.
Geldanamycin and its derivatives were evaluated for cytotoxicity activity against Vero, MCF-7, HeLa, and HepG2 cell lines using the MTT assay. Compounds $\mathbf{2}$ and $\mathbf{3}$ exhibited weak cytotoxicity activity toward Vero and HeLa cells with $\mathrm{IC}_{50}$ values of $>200.00 \mu \mathrm{g} / \mathrm{ml}$ (Table 5 and Fig. 4). However, these compounds were able to exhibit stronger cytotoxicity activity to MCF-7 and HepG2 than geldanamycin. These data were of interest, as it suggested that two novel geldanamycin derivatives were more toxic to some cancer cells than normal cells. Accordingly, they could be considered potential anticancer drugs in appropriate dosages. 
Taechowisan et al. / Journal of Applied Pharmaceutical Science 10 (06); 2020: 012-021

Table 2. Comparison of the spectral data of the compound $\mathbf{2}$ and compound $\mathbf{3}$.

\begin{tabular}{|c|c|c|c|c|}
\hline No. & $\delta_{\mathrm{C}}$ compound 2 & $\delta_{\mathrm{C}}$ compound 3 & $\delta_{\mathrm{H}}$ compound 2 & $\delta_{\mathrm{H}}$ compound 3 \\
\hline 1 & $168.4 \mathrm{C}$ & $168.4 \mathrm{C}$ & - & - \\
\hline 2 & $135.0 \mathrm{C}$ & $135.0 \mathrm{C}$ & - & - \\
\hline 2-Me & $12.5 \mathrm{CH}_{3}$ & $12.6 \mathrm{CH}_{3}$ & $2.02 \mathrm{~s}$ & $2.02 \mathrm{~s}$ \\
\hline 3 & $126.9 \mathrm{CH}$ & $127.0 \mathrm{CH}$ & $6.95 \mathrm{~d}(12)$ & $6.95 \mathrm{~d}(11.4)$ \\
\hline 4 & $126.5 \mathrm{CH}$ & $126.6 \mathrm{CH}$ & $6.58 \mathrm{t}(12)$ & $6.57 \mathrm{t}(11.4)$ \\
\hline 5 & $135.8 \mathrm{CH}$ & $138.7 \mathrm{CH}$ & $5.86 \mathrm{~m}$ & $5.86 \mathrm{~m}$ \\
\hline 6 & $81.2 \mathrm{CH}$ & $81.3 \mathrm{CH}$ & $4.30 \mathrm{~d}(9.9)$ & $4.31 \mathrm{~d}(9.9)$ \\
\hline 6-OMe & $57.1 \mathrm{CH}_{3}$ & $57.1 \mathrm{CH}_{3}$ & $3.26 \mathrm{~s}$ & $3.27 \mathrm{~s}$ \\
\hline 7 & $81.7 \mathrm{CH}$ & $81.1 \mathrm{CH}$ & $5.18 \mathrm{~s}$ & $5.18 \mathrm{~s}$ \\
\hline 7-OCONH & $156.1 \mathrm{C}$ & $156.1 \mathrm{C}$ & - & - \\
\hline 8 & $132.7 \mathrm{C}$ & $132.8 \mathrm{C}$ & - & - \\
\hline 8-Me & $12.7 \mathrm{CH}_{3}$ & $12.8 \mathrm{CH}_{3}$ & $1.80 \mathrm{~s}$ & $1.80 \mathrm{~s}$ \\
\hline 9 & $133.8 \mathrm{CH}$ & $133.8 \mathrm{CH}$ & $5.89 \mathrm{~m}$ & 5.89 \\
\hline 10 & $32.3 \mathrm{CH}$ & $32.4 \mathrm{CH}$ & $2.74 \mathrm{~m}$ & $2.74 \mathrm{~m}$ \\
\hline $10-\mathrm{Me}$ & $12.3 \mathrm{CH}_{3}$ & $12.4 \mathrm{CH}_{3}$ & $0.99 \mathrm{~d}(6.9)$ & $1.00 \mathrm{~d}(6.9)$ \\
\hline 11 & $72.6 \mathrm{CH}$ & $72.7 \mathrm{CH}$ & $3.57 \mathrm{~d}(9)$ & $3.57 \mathrm{~m}$ \\
\hline $11-\mathrm{OH}$ & - & - & - & - \\
\hline 12 & $81.5 \mathrm{CH}$ & $81.6 \mathrm{CH}$ & $3.45 \mathrm{~m}$ & $3.45 \mathrm{~m}$ \\
\hline 12-OMe & $56.7 \mathrm{OCH}_{3}$ & $56.7 \mathrm{OCH}_{3}$ & $3.36 \mathrm{~s}$ & $3.36 \mathrm{~s}$ \\
\hline 13 & $35.0 \mathrm{CH}_{2}$ & $35.2 \mathrm{CH}_{2}$ & $1.77 \mathrm{~m}$ & $1.77 \mathrm{~m}$ \\
\hline 14 & $28.5 \mathrm{CH}$ & $28.6 \mathrm{CH}$ & $1.77 \mathrm{~m}$ & $1.77 \mathrm{~m}$ \\
\hline 14-Me & $22.8 \mathrm{CH}_{3}$ & $23.0 \mathrm{CH}_{3}$ & $0.93 \mathrm{~d}(6.3)$ & $0.94 \mathrm{~d}(6.3)$ \\
\hline $15 \mathrm{a}$ & $34.4 \mathrm{CH}_{2}$ & $34.5 \mathrm{CH}_{2}$ & 2.70 & $2.68 \mathrm{~m}(12.5,9.9)$ \\
\hline $15 b$ & & & 2.40 & $2.44 \mathrm{~m}(12.5,4.8)$ \\
\hline 16 & $108.6 \mathrm{C}$ & $108.5 \mathrm{C}$ & - & - \\
\hline 17 & $144.9 \mathrm{C}$ & $145.0 \mathrm{C}$ & - & - \\
\hline 17-OMe & - & - & - & - \\
\hline 18 & $183.8 \mathrm{C}$ & $183.9 \mathrm{C}$ & - & - \\
\hline 19 & $108.7 \mathrm{CH}$ & $108.7 \mathrm{CH}$ & $7.24 \mathrm{~s}$ & $7.24 \mathrm{~s}$ \\
\hline 20 & $141.4 \mathrm{C}$ & $141.4 \mathrm{C}$ & - & - \\
\hline 21 & $180.5 \mathrm{C}$ & $180.6 \mathrm{C}$ & - & - \\
\hline $22 \mathrm{a}$ & $45.7 \mathrm{CH}_{2}$ & $45.6 \mathrm{CH}_{2}$ & $3.91 \mathrm{~m}$ & $3.92 \mathrm{~m}$ \\
\hline $22 b$ & & & $3.77 \mathrm{~m}$ & $3.76 \mathrm{~m}$ \\
\hline 23 & $25.75 \mathrm{CH}_{2}$ & $25.8 \mathrm{CH}_{2}$ & $3.15 \mathrm{t}(6.6)$ & $3.11 \mathrm{t}(6.6)$ \\
\hline 24 & $111.3 \mathrm{C}$ & $111.0 \mathrm{C}$ & - & - \\
\hline 25 & $122.5 \mathrm{CH}$ & $123.4 \mathrm{CH}$ & $7.13 \mathrm{~m}$ & 7.09 \\
\hline 26 & $136.6 \mathrm{C}$ & $131.8 \mathrm{C}$ & - & - \\
\hline 27 & $126.8 \mathrm{C}$ & $127.3 \mathrm{C}$ & - & - \\
\hline 28 & $111.5 \mathrm{CH}$ & $100.4 \mathrm{CH}$ & $7.40 \mathrm{~d}(7.8)$ & $7.00 \mathrm{~s}$ \\
\hline 29 & $119.8 \mathrm{CH}$ & $154.3 \mathrm{C}$ & $7.13 \mathrm{~m}$ & - \\
\hline 29-OMe & - & $56.0 \mathrm{OCH}_{3}$ & - & $3.87 \mathrm{~s}$ \\
\hline 30 & $125.6 \mathrm{CH}$ & $112.3 \mathrm{CH}$ & $7.15 \mathrm{~m}$ & $7.29 \mathrm{~d}(9)$ \\
\hline 31 & $118.5 \mathrm{CH}$ & $112.7 \mathrm{CH}$ & $7.60 \mathrm{~d}(7.8)$ & $6.90 \mathrm{~d}(9)$ \\
\hline $1-\mathrm{NH}$ & - & - & $9.17 \mathrm{~s}$ & $9.17 \mathrm{~s}$ \\
\hline $22-\mathrm{NH}$ & - & - & 6.47 brs $(6.0)$ & $6.47 \mathrm{t}(6.0)$ \\
\hline $25-\mathrm{NH}$ & - & - & $8.25 \mathrm{~s}$ & $8.14 \mathrm{~s}$ \\
\hline
\end{tabular}


Table 3. Comparison of the spectral data of the compound $\mathbf{3}$ and compound $\mathbf{1}$.

\begin{tabular}{|c|c|c|c|c|c|c|}
\hline No. & $\delta_{\mathrm{C}}$ compound 3 & $\delta_{\mathrm{C}}$ compound 1 & $\delta_{\mathrm{H}}$ compound 3 & $\delta_{\mathrm{H}}$ compound 1 & HMBC $(\mathrm{H} \rightarrow \mathrm{C})$ & COSY \\
\hline 1 & $168.4 \mathrm{C}$ & $169.7 \mathrm{C}$ & - & - & - & - \\
\hline 2 & $135.0 \mathrm{C}$ & $133.2 \mathrm{C}$ & - & - & - & - \\
\hline 2-Me & $12.6 \mathrm{CH}_{3}$ & $12.8 \mathrm{CH}_{3}$ & $2.02 \mathrm{~s}$ & $1.93 \mathrm{~s}$ & $1,2,3$ & - \\
\hline 3 & $127.0 \mathrm{CH}$ & $128.7 \mathrm{CH}$ & $6.95 \mathrm{~d}(11.4)$ & $6.95 \mathrm{~d}$ & $1,2,2-\mathrm{Me}, 4,5$ & 4 \\
\hline 4 & $126.6 \mathrm{CH}$ & $126.3 \mathrm{CH}$ & $6.57 \mathrm{t}(11.4)$ & $6.58 \mathrm{t}$ & $2,3,5$ & 3,5 \\
\hline 5 & $135.8 \mathrm{CH}$ & $138.7 \mathrm{CH}$ & $5.86 \mathrm{~m}$ & 5.81 brs & $3,4,6,7$ & 4,6 \\
\hline 6 & $81.3 \mathrm{CH}$ & $82.3 \mathrm{CH}$ & $4.31 \mathrm{~d}(9.9)$ & $4.36 \mathrm{~d}(7.6)$ & 4, 6-OMe & 5,7 \\
\hline 6-OMe & $57.1 \mathrm{CH}_{3}$ & $57.1 \mathrm{CH}_{3}$ & $3.27 \mathrm{~s}$ & $3.24 \mathrm{~s}$ & 6 & - \\
\hline 7 & $81.8 \mathrm{CH}$ & $81.1 \mathrm{CH}$ & $5.18 \mathrm{~s}$ & $4.88 \mathrm{brs}$ & $5,7-\mathrm{OCONH}_{2}, 8,8-\mathrm{Me}, 9$ & 6 \\
\hline 7-OCONH ${ }_{2}$ & $156.1 \mathrm{C}$ & $156.6 \mathrm{C}$ & - & - & - & - \\
\hline 8 & $132.8 \mathrm{C}$ & $129.1 \mathrm{C}$ & - & - & - & - \\
\hline 8-Me & $12.8 \mathrm{CH}_{3}$ & $13.0 \mathrm{CH}_{3}$ & $1.80 \mathrm{~s}$ & $1.62 \mathrm{~s}$ & $7,8,9$ & - \\
\hline 9 & $133.8 \mathrm{CH}$ & $132.4 \mathrm{CH}$ & $5.89 *$ & $5.50 \mathrm{~d}(8.5)$ & 7, 8-Me, 10-Me, 11 & 10 \\
\hline 10 & $32.4 \mathrm{CH}$ & $32.6 \mathrm{CH}$ & $2.74 \mathrm{~m}$ & 2.56 & $8,9,10-\mathrm{Me}$ & $9,10-\mathrm{Me}, 11$ \\
\hline $10-\mathrm{Me}$ & $12.4 \mathrm{CH}_{3}$ & $13.4 \mathrm{CH}_{3}$ & $1.00 \mathrm{~d}(6.9)$ & $0.75 \mathrm{~d}(6.8)$ & $9,10,11$ & 10 \\
\hline 11 & $72.7 \mathrm{CH}$ & $72.4 \mathrm{CH}$ & $3.57 \mathrm{~m}$ & 3.09 brs & $9,10,10-\mathrm{Me}, 12$ & 10,12 \\
\hline $11-\mathrm{OH}$ & - & - & - & - & - & - \\
\hline 12 & $81.6 \mathrm{CH}$ & $80.7 \mathrm{CH}$ & $3.45 \mathrm{~m}$ & $3.09 \mathrm{brs}$ & $10,11,12-\mathrm{OMe}, 14$ & 11,13 \\
\hline $12-\mathrm{OMe}$ & $56.7 \mathrm{OCH}_{3}$ & $56.5 \mathrm{CH}_{3}$ & $3.36 \mathrm{~s}$ & $3.23 \mathrm{~s}$ & 12 & - \\
\hline 13 & $35.2 \mathrm{CH}_{2}$ & $31.3 \mathrm{CH}_{2}$ & $1.77 \mathrm{~m}$ & $1.45 \mathrm{brs}$ & $11,12,14$ & 12 \\
\hline 14 & $28.6 \mathrm{CH}$ & $27.1 \mathrm{CH}$ & $1.77 \mathrm{~m}$ & $1.93 \mathrm{~s}$ & 12,13 & $14-\mathrm{Me}, 15 \mathrm{~b}$ \\
\hline $14-\mathrm{Me}$ & $23.0 \mathrm{CH}_{3}$ & $23.9 \mathrm{CH}_{3}$ & $0.94 \mathrm{~d}(6.3)$ & 0.97 brs & $13,14,15$ & 14 \\
\hline $15 \mathrm{a}$ & $34.5 \mathrm{CH}_{2}$ & $32.2 \mathrm{CH}_{2}$ & $2.68 \mathrm{~m}$ & 2.43 dd $(12.5,9.9)$ & 13, 14, 14-Me, 16, 17, 21 & $15 \mathrm{~b}$ \\
\hline $15 b$ & & & $2.44 \mathrm{~m}$ & $2.18 \mathrm{dd}(12.5,4.8)$ & $14,16,17,21$ & $14,15 \mathrm{a}$ \\
\hline 16 & $108.5 \mathrm{C}$ & $128.7 \mathrm{C}$ & - & - & - & - \\
\hline 17 & $145.0 \mathrm{C}$ & $156.9 \mathrm{C}$ & - & - & - & - \\
\hline 17-OMe & - & $61.6 \mathrm{CH}_{3}$ & - & $3.96 \mathrm{~s}$ & - & - \\
\hline 18 & $183.9 \mathrm{C}$ & $184.3 \mathrm{C}$ & - & - & - & - \\
\hline 19 & $108.7 \mathrm{CH}$ & $111.3 \mathrm{CH}$ & $7.24 \mathrm{~s}$ & $7.04 \mathrm{~s}$ & 17,21 & - \\
\hline 20 & $141.4 \mathrm{C}$ & $140.1 \mathrm{C}$ & - & - & - & - \\
\hline 21 & $180.6 \mathrm{C}$ & $183.6 \mathrm{C}$ & - & - & - & - \\
\hline $22 \mathrm{a}$ & $45.6 \mathrm{CH}_{2}$ & - & $3.92 \mathrm{~m}$ & - & 23,24 & $22 \mathrm{~b}, 22-\mathrm{NH}, 23$ \\
\hline $22 \mathrm{~b}$ & & & $3.76 \mathrm{~m}$ & - & 23,24 & $22 \mathrm{a}, 22-\mathrm{NH}, 23$ \\
\hline 23 & $25.8 \mathrm{CH}_{2}$ & - & $3.11 \mathrm{t}(6.6)$ & - & $22,24,25$ & $22 \mathrm{a}, 22 \mathrm{~b}$ \\
\hline 24 & $111.0 \mathrm{C}$ & - & - & - & - & - \\
\hline 25 & $123.4 \mathrm{CH}$ & - & $7.09 *$ & - & $23,24,26,27$ & - \\
\hline 26 & $131.8 \mathrm{C}$ & - & - & - & - & - \\
\hline 27 & $127.3 \mathrm{C}$ & - & - & - & - & - \\
\hline 28 & $100.4 \mathrm{CH}$ & - & $7.00 \mathrm{~s}$ & - & $24,26,29,30$ & - \\
\hline 29 & $154.3 \mathrm{C}$ & - & - & - & - & - \\
\hline 29-OMe & $56.0 \mathrm{OCH}_{3}$ & - & $3.87 \mathrm{~s}$ & - & 29 & - \\
\hline 30 & $112.3 \mathrm{CH}$ & - & $7.29 \mathrm{~d}(9)$ & - & 29 & 31 \\
\hline 31 & $112.7 \mathrm{CH}$ & - & $6.90 \mathrm{~d}(9)$ & - & $26,27,29$ & 30 \\
\hline $1-\mathrm{NH}$ & - & - & $9.17 \mathrm{~s}$ & $9.18, \mathrm{NH}$, brs & $1,19,21$ & - \\
\hline $22-\mathrm{NH}$ & - & - & $6.47 \mathrm{t}(6.0)$ & - & $16,18,22,23$ & $22 \mathrm{a}, 22 \mathrm{~b}$ \\
\hline $25-\mathrm{NH}$ & - & - & $8.14 \mathrm{~s}$ & - & $24,25,26,27$ & 25 \\
\hline
\end{tabular}



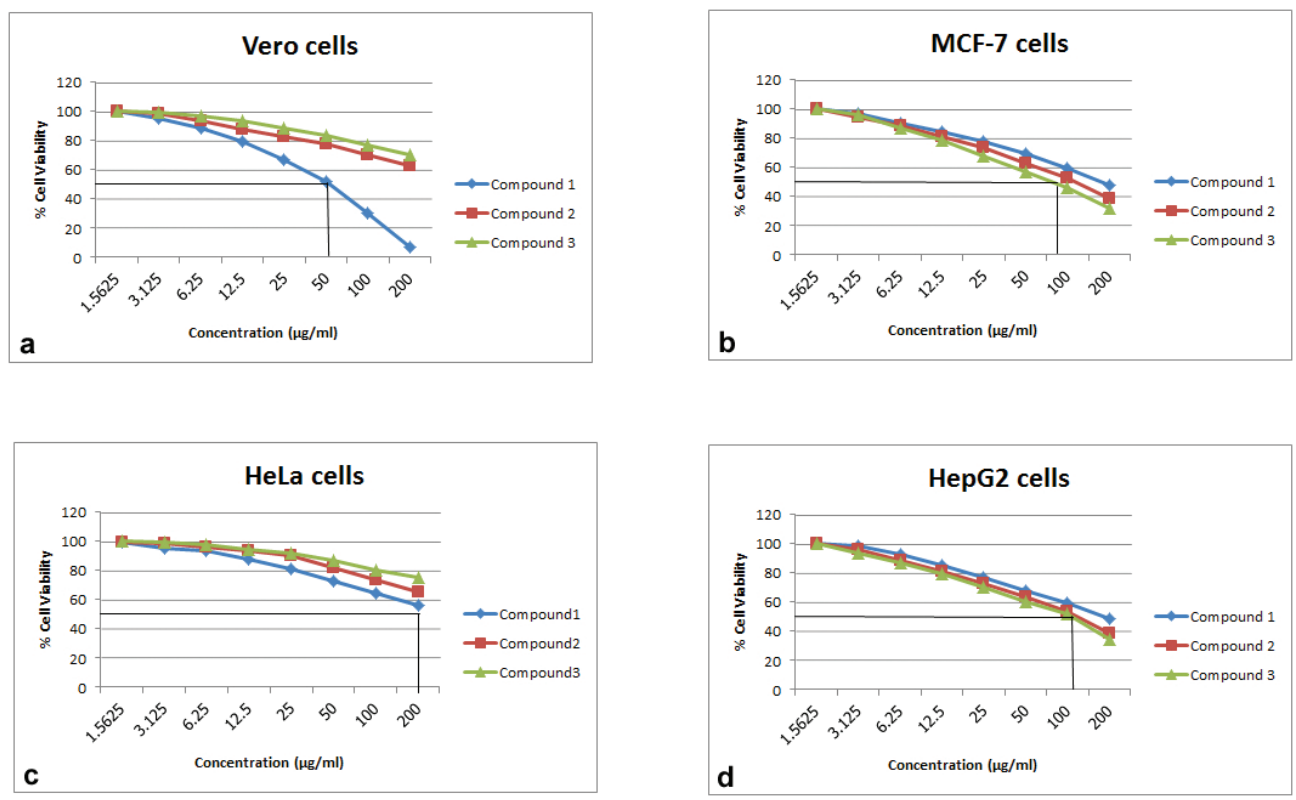

Figure 4. Cytotoxicity effects of compounds $1(\bullet), 2(\bullet)$ and 3(A) on Vero cells (a), MCF-7 cells (b), HeLa cells (c) and HepG2 cells (d) using an MTT colorimetric assay.

Table 4. Water solubility of geldanamycin (1) and its derivatives (2 and $\mathbf{3})$.

\begin{tabular}{|c|c|c|c|c|}
\hline Compounds & MW & Solubility in water $(\mathrm{mg} / \mathrm{ml})^{\mathrm{b}}$ & Solubility in water $(\mu M)^{a}$ & Relative solubility \\
\hline 1 & 560 & $0.085 \pm 0.004$ & $151.78 \pm 7.14^{\mathrm{b}}$ & 1.00 \\
\hline 2 & 688 & $0.200 \pm 0.003$ & $290.69 \pm 4.36^{\mathrm{c}}$ & 1.91 \\
\hline 3 & 718 & $0.250 \pm 0.003$ & $348.18 \pm 5.02^{\mathrm{d}}$ & 2.29 \\
\hline
\end{tabular}

aThe results presented represent the average of three separate experiments (mean $\pm \mathrm{SD}$ ).

b,c,dSignificant differences $(p<0.05)$.

Table 5. Cytotoxicity activity $\left(\mathrm{IC}_{50}\right)$ of geldanamycin (1) and its derivatives (2 and $\left.\mathbf{3}\right)$.

\begin{tabular}{cccccccc}
\hline \multirow{2}{*}{ Compounds } & \multicolumn{4}{c}{ IC50a $(\boldsymbol{\mu g} / \mathbf{m l})$} & \multicolumn{3}{c}{ Therapeutic index $^{\mathrm{b}}$} \\
\cline { 2 - 7 } & Vero $^{\mathrm{c}}$ & MCF-7 & HeLa & HepG2 & MCF-7 & HeLa & HepG2 \\
\hline $\mathbf{1}$ & 54.25 & 178.43 & $>200.00$ & 184.92 & 0.30 & ND $^{\mathrm{i}}$ & 0.11 \\
$\mathbf{2}$ & $>200.00^{\mathrm{d}}$ & $105.62^{\mathrm{e}}$ & $>200.00$ & $124.57^{\mathrm{f}}$ & $>1.90^{\mathrm{g}}$ & ND & $>1.60^{\mathrm{h}}$ \\
$\mathbf{3}$ & $>200.00^{\mathrm{d}}$ & $82.50^{\mathrm{c}}$ & $>200.00$ & $114.35^{\mathrm{f}}$ & $>2.42^{\mathrm{g}}$ & ND & $>1.75^{\mathrm{h}}$ \\
\hline
\end{tabular}

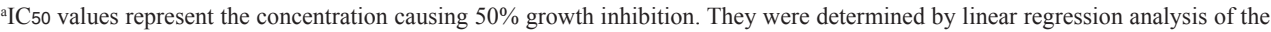
average of three separate experiments.

${ }^{b}$ Therapeutic index is defined as the ratio of the median toxic dose on normal cells to the median effective dose on cancer cells.

${ }^{c}$ Vero $=$ African green monkey kidney cell line, MCF-7 = human breast carcinoma cell line, HeLa = human cervical carcinoma cell line, HepG2 = human hepatocellular carcinoma cell line.

d,e,f,g,h Significant differences from the compound $\mathbf{1}(p<0.05)$.

${ }^{\mathrm{i}} \mathrm{ND}=$ not determined.

\section{DISCUSSION}

The benzoquinone ansamycins are an important class of Hsp90 inhibitors that possess potent anticancer activity in preclinical models and may emerge as efficacious therapeutic agents for the treatment of cancer (Isaacs, 2005). More generally, Hsp90 is an attractive therapeutic anticancer target because inhibition of its chaperone activity results in lower cellular levels of multiple client proteins that are critical for cancer-cell survival (Neckers, 2002). Geldanamycin, an ansamycin antibiotic, along with its analogues, had significant anticancer properties (Schnur et al., 1995; Whitesell et al., 1992). These compounds bind specifically to the adinosine triphosphate (ATP) binding site of Hsp90 agents, these disrupted Hsp90 association with client protein (Grenert et al., 1997; Prodromou et al., 1997; Stebbins et al., 1997). Therefore, the prevention of binding of Hsp90 with ATP affected extremely the composition of Hsp90-containing chaperone complexes (An et al., 1997; Obermann etal., 1998; Workman et al., 2002). However, the use of geldanamycin and its derivatives as a chemotherapeutic agent did not proceed owing to its severe hepatotoxicity, metabolic instability, and poor water solubility (Fukuyo et al., 2009; Supko et al., 1995). The improvement of water solubility leads to drug potency and advantageous pharmacological profiles. Therefore, water-soluble geldanamycin derivatives with improved pharmacokinetic and pharmacological properties are necessary (Cheng et al., 2005; Wu et al., 2012). Tryptamine has been an effective tool for improving the water solubility, biological potency, and pharmacokinetic properties of many natural products (Kousara et al., 2017). Recently, tryptamine derivatives have showed moderate to good anticancer 
activity against HepG2, HeLa, CNE1, and A549 human cancer cell lines with $\mathrm{IC}_{50}$ values of 16.5-18.7 $\mu \mathrm{M}$ (Guo et al., 2019). In this study, geldanamycin exhibited lower solubility in water than its derivatives. It also exhibited more cytotoxicity against normal cells than cancer cells, but no cytotoxicity against HeLa cells. This differs from those reports that geldanamycin was extremely active against $\mathrm{KB}$ cells, an ubiquitous keratin-forming HeLa cell line $(<0.001 \mu \mathrm{g} /$ $\mathrm{ml}$ ) (Deboer et al., 1970), but showed moderate cytotoxicity against MCF-7 and HepG2. According to other reports, geldanamycin was active against various human breast cancer cell lines including: $\mathrm{SKBr} 3\left(\mathrm{IC}_{50}\right.$ value of $\left.37 \mathrm{nM}\right)$ (Hu et al., 2004); BC ( $\mathrm{IC}_{50}$ value of $0.01 \mu \mathrm{g} / \mathrm{ml}$ ) (Jongrungruangchok et al., 2006); MDA-MB-231, MCF-7 and T-47D ( $\mathrm{IC}_{50}$ value of $\left.100 \mu \mathrm{M}\right)$ and human hepatocellular carcinoma cell lines, HepG2, and SMMC7721 ( $\mathrm{IC}_{50}$ value of 200 $\mu \mathrm{M})$ (Zhang et al., 2016). In contrast, cytotoxicity activity of tryptaminated geldanamycins against cancer cells (MCF-7 and HepG2) exhibited greater activity than geldanamycin. This was due to the increased water solubility of geldanamycin derivatives and enhanced anticancer activity of tryptaminated geldanamycins.

\section{CONCLUSION}

In summary, the two new tryptaminated-geldanamycins have been synthesized; they showed the water solubility improvement. They also have potent cytotoxic effects on MCF-7; human breast cancer cells, and HepG2 with low cytotoxicity against Vero cells. These results suggest that the tryptaminated-geldanamycin derivatives may be a potential therapeutic candidate for the treatment of some cancers. They could be useful for future drug development.

\section{ACKNOWLEDGMENTS}

The authors are grateful to Ms Chanjira Jaramornburapong and Ms Chanakan Winyakul in the Department of Chemistry, Faculty of Science, Silpakorn University, Thailand, for measuring NMR and MS data, respectively. This work was supported by the Faculty of Science, Silpakorn University, Nakhon Pathom, Thailand.

\section{CONFLICT OF INTEREST}

Authors declare that they do not have any conflicts of interest.

\section{REFERENCES}

An WG, Schnur RC, Neckers L, Blagosklonny MV. Depletion of $\mathrm{p} 185 \mathrm{erbB} 2$, Raf-1, and mutant $\mathrm{p} 53$ proteins by geldanamycin derivatives correlates with antiproliferative activity. Cancer Chemother Pharm, 1997; 40(1):60-4.

Cheng H, Cao X, Xian M, Fang L, Cai TB, Ji JJ, Tunac JB, Sun D, Wang PG. Synthesis and enzyme-specific activation of carbohydrategeldanamycin conjugates with potent anticancer activity. J Med Chem, 2005; 48(2):645-52.

Coombs JT, Franco CMM. Isolation and identification of actinobacteria from surface-sterilized wheat roots. Appl Environ Microbiol, 2003; 69(9):5603-8.

Deboer C, Meulman PA, Wnuk RJ, Peterson DH. Geldanamycin, a new antibiotic. J Antibiot, 1970; 23(9):442-7.

Fukuyo Y, Hunt CR, Horikoshi N. Geldanamycin and its anticancer activities. Cancer Lett, 2009; 290(1):24-35.

Grenert JP, Sullivan WP, Fadden P, Haystead TA, Clark J, Mimnaugh E, Krutzsch H, Ochel HJ, Schulte TW, Sausville E, Neckers LM, Toft DO. The amino-terminal domain of heat shock protein 90 (Hsp90) that binds geldanamycin is an ATP/ADP switch domain that regulates Hsp90 conformation. J Biol Chem, 1997; 272(38):23843-50.

Guo Z, Xu Y, Peng Y, Rashid HU, Quan W, Xie P, Wu L, Jiang J, Wang L, Liu X. Design, synthesis and evaluation of novel (S)-tryptamine derivatives containing an allyl group and an aryl sulfonamide unit as anticancer agents. Bioorg Med Chem Lett, 2019; 29(1):1133-7.

Hoelke B, Gieringer S, Arlt M, Saal C. Comparison of nephelometric, UV-spectroscopic, and HPLC methods for high-throughput determination of aqueous drug solubility in microtiter plates. Anal Chem, 2009; 81(8):3165-72.

Hu Z, Liu Y, Tian ZQ, Ma W, Starks CM, Regentin R, Licari $\mathrm{P}$, Myles DC, Hutchinson CR. Isolation and characterization of novel geldanamycin analogues. J Antibiot, 2004; 57(7):421-8.

Isaacs JS. Heat-shock protein 90 inhibitors in antineoplastic therapy: is it all wrapped up? Expert Opin Investig Drugs, 2005; 14(6) 569-89.

Jongrungruangchok S, Tanasupawat S, Kittakoop P, Bavovada R, Kobayashi H, Kudo T. Identification of Streptomyces and Kitasatospora strains from Thai soils with geldanamycin production strain. Actinomycetologica, 2006; 20(1):10-14.

Jurczyszyn A, Zebzda A, Czepiel J, Perucki W, Banzan-Socha S, Cibor D, Owczarek D, Majka M. Geldanamycin and its derivatives inhibit the growth of myeloma cells and reduce the expression of the MET receptor. J Cancer, 2014; 5(6):480-90.

Kousara S, Anjuma SN, Jaleela F, Khana J, Naseema S. Biomedical significance of tryptamine: a review. J Pharmacovigil, 2017; 5(5):239.

Le Brazidec JY, Kamal A, Busch D, Thao L, Zhang L, Timony G, Grecko R, Trent K, Lough R, Salazar T, Khan S, Burrows F, Boehm MF. Synthesis and biological evaluation of a new class of geldanamycin derivatives as potent inhibitors of Hsp90. J Med Chem, 2004; 47(15): 3865-73.

Lee EJ. Cancer chemoprevention effects of geldanamycin and 17-AAG in human oral squamous cell carcinoma. Korean J Clin Lab Sci, 2018; 50(4):462-9.

Lin Z, Peng R, Li Z, Wang Y, Lu C, Shen Y, Wang J, Shi G. 17ABAG, a novel geldanamycin derivative, inhibits $\mathrm{LNCaP}$ cell proliferation through heat shock protein 90 inhibition. Int J Mol Med, 2015; 36(2) 424-32.

Malik MA, Raza MK, Dar OA, Amadudin, Abid M, Wani MY, Al-Bogami AS, Hashmi AA. Probing the antibacterial and anticancer potential of tryptamine based mixed ligand schiff base ruthenium(III) complexes. Bioorg Chem, 2019; 87:773-82.

Mimnaugh EG, Chavany C, Neckers L. Polyubiquitination and proteasomal degradation of the $185 \mathrm{c}$-erbB-2 receptor protein-tyrosine kinase induced by geldanamycin. J Biol Chem, 1996; 271(37):22796-801.

Modi S, Stopeck A, Linden H, Solit D, Chandarlapaty S, Rosen N, D'Andrea G, Dickler M, Moynahan ME, Sugarman S, Ma W, Patil S, Norton L, Hannah AL, Hudis C. HSP90 inhibition is effective in breast cancer: a phase II trial of tanespimycin (17-AAG) plus trastuzumab in patients with HER2-positive metastatic breast cancer progressing on trastuzumab. Clin Cancer Res, 2011; 17(15):5132-9.

Neckers L. Hsp90 inhibitors as novel cancer chemotherapeutic agents. Trends Mol Med, 2002; 8(4):S55-61.

Obermann WM, Sondermann H, Russo AA, Pavletich NP, Hartl FU. In vivo function of HSP90 is dependent on ATP binding and ATP hydrolysis. J Cell Biol, 1998; 143(4):901-10.

Ōmura S, Nakagawa A, Sadakane N. Structure of herbimycin a new ansamycin antibiotic. Tetrahedron Lett, 1979; 20(44):4323-6.

Prodromou C, Roe SM, O’Brien R, Ladbury JE, Piper PW, Pearl LH. Identification and structural characterization of the ATP/ADP-binding site in the Hsp90 molecular chaperone. Cell, 1997; 90(1):65-75.

Qin HL, Panek JS. Total synthesis of the Hsp90 inhibitor geldanamycin. Organic Lett, 2008; 10(12):2477-9.

Schnur RC, Corman ML, Gallaschun RJ, Cooper BA, Dee MF, Doty JL, Muzzi ML, Moyer JD, DiOrio CI, Barbacci EG, Miller PE, Pollalk VA, Savage DM, Sloan DE, Pustilnik LR, Moyer MP. ErbB-2 oncogene inhibition by geldanamycin derivatives: synthesis, mechanism of action, and structure-activity relationships. J Med Chem, 1995; 38(19):3806-12.

Stebbins CE, Russo AA, Schneider C, Rosen N, Hartl FU, Pavletich NP. Crystal structure of an Hsp90-geldanamycin complex: 
targeting of a protein chaperone by an antitumor agent. Cell, 1997; 89(2):239-50.

Supko JG, Hickman RL, Grever MR, Malspeis L. Preclinical pharmacologic evaluation of geldanamycin as an antitumor agent. Cancer Chemoth Pharm, 1995; 36(4):305-15.

Taechowisan T, Chaisaeng S, Phutdhawong WS. Antibacterial, antioxidant and anticancer activities of biphenyls from Streptomyces sp. BO-07; an endophyte in Boesenbergia rotunda (L.) Mansf A. Food Agri Immunol, 2017; 28(6):1330-46.

Taechowisan T, Lumyong S. Activity of endophytic actinomycetes from roots of Zingiber officinale and Alpinia galanga against phytopathogenic fungi. Ann Microbiol, 2003; 53(3): 291-8.

Taechowisan T, Puckdee W, Phutdhawong WS. Streptomyces zerumbet, a Novel species from Zingiber zerumbet (L.) Smith and isolation of its bioactive compounds. Adv Microbiol, 2019; 9(3):194-219.

Tian ZQ, Liu Y, Zhang D, Wang Z, Dong SD, Carreras CW, Zhou Y, Rastelli G, Santi DV, Myles DC. Synthesis and biological activities of novel 17-aminogeldanamycin derivatives. Bioorg Med Chem, 2004; 12(20):5317-29.

Vasilevskaya IA, Rakitina TV, O’Dwyer PJ. Geldanamycin and its 17-allylamino-17-demethoxy analogue antagonize the action of cisplatin in human colon adenocarcinoma cells: differential caspase activation as a basis for interaction. Cancer Res, 2003; 63(12):3241-6.

Wang X, Su H, Wang W, Chen C, Cao X. Peptidomimetics based on dehydroepiandrosterone scaffold: synthesis, antiproliferation activity, structure-activity relationship, and mechanisms. Sci Rep, 2016; 6:32654.

Whitesell L, Mimnaugh EG, De Costa B, Myers CE, Neckers LM. Inhibition of heat shock protein HSP90-pp60v-src heteroprotein complex formation by benzoquinone ansamycins: essential role for stress proteins in oncogenic transformation. Proc Natl Acad Sci USA, 1994; 91(18):8324-8.
Whitesell L, Shifrin SD, Schwab G, Neckers LM. Benzoquinonoid ansamycins possess selective tumoricidal activity unrelated to src kinase inhibition. Cancer Res, 1992; 52(7):1721-8.

Workman P, Maloney A. HSP90 as a new therapeutic target for cancer therapy: the story unfolds. Expert Opin Biol Ther, 2002; 2(1):3-24.

Wrona IE, Gozman A, Taldone T, Chiosis, G, Panek JS. Synthesis of reblastatin, autolytimycin, and non-benzoquinone analogues: potent inhibitors of heat shock protein 90. J Org Chem, 2010; 75(9):2820-35.

Wu CZ, Jang JH, Woo MH, Ahn JS, Kim JS, Hong YS. Enzymatic glycosylation of non benzoquinone geldanamycin analogs via Bacillus UDP-glycosyltransferase. Appl Environ Microbiol, 2012; 78(21):7680-6.

Zhang Z, Li HM, Zhou C, Li Q, Ma L, Zhang Z, Sun Y, Wang L, Zhang X, Zhu B, Hong YS, Wu CZ, Liu H. Non-benzoquinone geldanamycin analogs trigger various forms of death in human breast cancer cells. J Exp Clin Cancer Res, 2016; 35(1):149.

\section{How to cite this article:}

Taechowisan T, Samsawat T, Puckdee W, Phutdhawong WS. Cytotoxicity activity of geldanamycin derivatives against various cancer cell lines. J Appl Pharm Sci, 2020; 10(06):012-021. 